\title{
The role of the temporal lobe semantic system in number knowledge: evidence from late-stage semantic dementia
}

\author{
Elizabeth Jefferies $^{\mathrm{a}, *}$, David Bateman ${ }^{\mathrm{b}}$, Matthew A. Lambon Ralph ${ }^{\mathrm{a}}$

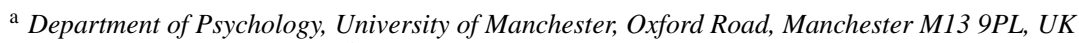 \\ ${ }^{\mathrm{b}}$ Royal United Hospital, Bath, UK
}

Received 6 February 2004; received in revised form 10 September 2004; accepted 21 September 2004

\begin{abstract}
Previous reports have demonstrated that many aspects of number knowledge remain unimpaired in semantic dementia, despite severe comprehension problems in other domains. It is argued that this advantage for numbers arises because the disease spares the parietal lobe magnitude system thought to be critical for number processing. Models of numerical cognition that favour a separation between verbal and magnitude representations of number might, however, predict a restricted impairment of the verbal number code in this condition. We obtained support for this hypothesis in a patient with late-stage semantic dementia. She was impaired at a variety of tasks tapping the verbal number code; for example, reading and writing Arabic numerals, naming and word-picture matching with dot pictures, reading aloud number words, digit span and magnitude comparison/serial ordering tasks with number words. In contrast, she demonstrated good understanding of the magnitude and serial order of numbers when tested with Arabic numerals and non-symbolic representations. These findings suggest that although the magnitude meaning of numbers is isolated from the temporal lobe semantic system, the anterior infero-temporal lobe may play a critical role in binding English number words to their non-symbolic magnitude meaning.
\end{abstract}

(C) 2004 Elsevier Ltd. All rights reserved.

Keywords: Number knowledge; Semantic dementia

\section{Introduction}

Several recent studies have found that number knowledge is relatively spared in patients with semantic dementia (SD), in contrast to the marked and progressive decline of non-numerical conceptual knowledge that is the hallmark of this condition (Cappelletti, Butterworth, \& Kopelman, 2001; Crutch \& Warrington, 2002; Diesfeldt, 1993; Jefferies, Patterson, Jones, Bateman, \& Lambon Ralph, 2004b). SD patients have impaired comprehension on a range of verbal and non-verbal tasks (Bozeat, Lambon Ralph, Patterson, Garrard, \& Hodges, 2000) and have increasing difficulty recognising objects and faces as the disease progresses. Their spontaneous speech is fluent but characterised by word finding difficulties. In picture naming they make

\footnotetext{
* Corresponding author. Tel.: +44 161275 7338; fax: +44 1612752588.

E-mail address: beth.jefferies@manchester.ac.uk (E. Jefferies).
}

semantic errors (bear $\rightarrow$ "horse") and superordinate errors (bear $\rightarrow$ "animal") as well as frequent omissions (Lambon Ralph, McClelland, Patterson, Galton, \& Hodges, 2001). However, other cognitive domains, including non-verbal reasoning, perceptual and spatial skills, new episodic learning, syntax and phonology, remain largely intact (Hodges, Patterson, Oxbury, \& Funnell, 1992; Snowden, Neary, \& Mann, 1996b).

The semantic impairment in SD typically affects every category of non-numerical knowledge (Lambon Ralph, Patterson, Garrard, \& Hodges, 2003), yet number comprehension is consistently reported to be spared. Comparison of the magnitude of numbers is typically found to be perfect. Patients also show good knowledge of the serial order of numbers (Cappelletti et al., 2001; Jefferies et al., 2004b). Every published case to date has shown a preserved ability to read and write Arabic numerals. In addition, SD patients have better reading and spelling of English number words 
than non-number words matched for regularity and frequency (Butterworth, Cappelletti, \& Kopelman, 2001; Cappelletti, Kopelman, \& Butterworth, 2002; Jefferies et al., 2004b). This finding is consistent with the view that semantic representations play an important role in reading aloud and spelling (Plaut, McClelland, Seidenberg, \& Patterson, 1996): the correct pronunciation/spelling of number words is thought to reflect the activation derived from these preserved concepts. Similarly, SD patients show better verbal short-term memory (STM) for numbers compared with non-number words (Jefferies et al., 2004b). This result mirrors the previously documented immediate serial recall (ISR) advantage for relatively well-known words over semantically degraded items (Jefferies, Jones, Bateman, \& Lambon Ralph, 2004a, in press; Knott, Patterson, \& Hodges, 1997, 2000) and is consistent with the theory that semantic representations make an important contribution to verbal STM (Martin \& Saffran, 1997; Patterson, Graham, \& Hodges, 1994). In everyday life, patients typically remain able to manage their money, use a diary and tell the time at least until a late stage of the disease. According to Snowden et al. (1996b), a significant proportion of patients become obsessional about numbers; they show compulsive counting, constant clock-watching or excessive thrift with money.

The relative preservation of number knowledge in SD is consistent with the view that numerals are separable to some extent at the semantic level. There is a double dissociation between the comprehension/production of numbers and other classes of stimuli in line with this standpoint (Anderson, Damasio, \& Damasio, 1990; Cipolotti, Butterworth, \& Denes, 1991; Rossor, Warrington, \& Cipolotti, 1995; Thioux et al., 1998). It has been suggested that number comprehension is relatively preserved in SD because the meaning of these stimuli is derived primarily from their magnitude (Cappelletti et al., 2001). The pathology underlying SD apparently spares the parietal areas thought to be critical for magnitude (Dehaene \& Cohen, 1995; Dehaene, DehaeneLambertz, \& Cohen, 1998). Damage to the inferior parietal lobe is associated with acalculia (e.g., Cipolotti et al., 1991; Dehaene \& Cohen, 1997; Delazer \& Benke, 1997; Takayama, Sugishita, Akiguchi, \& Kimura, 1994) and functional imaging studies of number and calculation tasks reveal activation in inferior parietal regions (Dehaene et al., 1996; Dehaene, Spelke, Pinel, Stanescu, \& Tsivkin, 1999; Pinel, Dehaene, Rivière, \& LeBihan, 2001; Stanescu-Cosson et al., 2000). In contrast, the cortical atrophy in SD predominantly affects the anterior and inferior temporal lobes bilaterally, and the temporal poles in particular (Galton et al., 2001; Mummery et al., 2000). Hence, the core magnitude meaning of number (which is typically termed "semantic" in models of numerical cognition: e.g., McCloskey, Caramazza, \& Basili, 1985) is independent of the temporal lobe semantic system.

Despite this preservation of number knowledge, some numerical skills do show a mild decline in SD. Although the ability to perform calculations is largely intact, knowledge of arithmetical procedures and signs can be impaired and errors frequently reflect the use of the wrong operation (Cappelletti et al., 2001; Jefferies et al., 2004b). Patients typically show greater difficulties on multiplication/division than other operations (Cappelletti et al., 2001; Jefferies et al., 2004b; Lemer, Dehaene, Spelke, \& Cohen, 2003) and some of their errors (e.g., $2 \times 3=8$ ) are indicative of a failure to retrieve previously familiar arithmetic facts (e.g., "two times three is six"). SD patients also have impaired knowledge of encyclopaedic and personal number facts (e.g., what is the boiling point of water? What is your shoe size?), in line with their more general semantic problems (Cappelletti et al., 2001).

At a behavioural level, this pattern of competencies and weaknesses in SD is broadly consistent with Dehaene and Cohen's 'triple code' model (Dehaene, 1992; Dehaene \& Cohen, 1995). According to this model, there is (1) an analogue representation of numerical magnitude in the parietal lobe that is separable from both (2) the verbal code for number words and previously learned arithmetic facts, and (3) the visual code for Arabic numerals. The parietal magnitude representation should be spared in SD, consistent with the observation that patients perform well in comparison tasks (although some authors have argued that linguistic representations do play a role in this task: e.g., Noel \& Seron, 1997). Other types of number processing (e.g., transcoding, retrieval of arithmetic facts), which purportedly draw more heavily on the verbal code, might show greater impairment in SD, in line with these patients' severe word finding difficulties.

Lemer et al. (2003) obtained specific support for the hypothesis that number words but not their corresponding magnitudes are degraded in SD. They observed an impairment of exact but not approximate calculation and also poorer multiplication than subtraction in a patient with SD. Exact arithmetic is thought to rely on a verbal number code, whereas approximate calculation is supposedly underpinned by magnitude manipulation (see Dehaene et al., 1998, for a review). Similarly, multiplication may typically be solved by retrieving memorised facts (Ashcraft, 1992) stored in a verbal code, whereas subtraction may be more reliant on quantity manipulation (Dehaene, 1992; Dehaene \& Cohen, 1995; Dehaene et al., 1999). Lemer et al. also obtained evidence of intact processing of non-symbolic numerosity in SD. Enumeration of one to three items was rapid suggesting normal subitizing (i.e., the ability to determine the number of items in an array at a glance, without counting) but reaction times were sharply increased for larger arrays, suggesting that counting was substantially slowed.

The triple code model makes explicit predictions about the anatomical organisation of number processing. As noted above, the analogue magnitude representation is thought to be underpinned by parietal areas; in particular the horizontal segment of the intraparietal sulcus (Dehaene, Piazza, Pinel, \& Cohen, 2003; Pinel et al., 2001). In contrast, the visual code for Arabic numerals is represented in the occipital-temporal regions of the ventral visual pathway bilaterally, while the verbal code is argued to draw on perisylvian language areas of the left hemisphere, including the left angular gyrus. All 
of these brain regions (even those supposedly underpinning the verbal number code) remain relatively intact in SD. The triple code model does not propose a role for anterior inferotemporal cortex in numerical cognition and so, on the face of it, cannot account for the circumscribed difficulties of SD patients in number tasks.

Although the temporal lobe semantic system has not been specifically implicated in previous models of numerical cognition, some patients show better reading of familiar numbers, such as famous dates and brands of car, compared with unfamiliar numbers (Cohen, Dehaene, \& Verstichel, 1994; Delazer \& Girelli, 1997). Similarly, normal participants show associative priming effects in digit naming (e.g., Boeing-747; Alameda, Cuetos, \& Brysbaert, 2003), demonstrating a role for lexical-semantic knowledge in number transcoding. The temporal lobe semantic system is thought to represent multi-modal mappings between different sensory inputs: e.g., visual and verbal (Rogers et al., 2004a). By extension, this region might be expected to underpin the connections between different number codes (parietal/magnitude, verbal and visual), with the consequence that, at a late stage of SD, number words are not recognised as referring to particular Arabic numerals and magnitudes. In this sense, the verbal number code should become impaired while knowledge of nonsymbolic magnitudes remains intact. SD patients have poor comprehension of both words and pictures (Bozeat et al., 2000) and so might also lose their knowledge of which Arabic numerals refer to specific magnitudes/number words. However, patients with more left than right-sided atrophy (such as the case presented here) might exhibit greater impairment of number words than Arabic numbers; a point we will return to later.

Although the evidence discussed so far is coherent with the view that SD produces specific degradation of number words but not their magnitudes, the typically observed pattern of preservation and impairment across tasks in SD (e.g., Butterworth et al., 2001; Cappelletti et al., 2001; Jefferies et al., 2004b) is not wholly predicted by the extent to which they draw on these two codes. SD patients have been found to have intact transcoding skills and show an advantage for number over non-number words in reading, spelling and verbal STM and yet these tasks draw heavily on the verbal number code which is thought to become degraded in this condition. One possible resolution of this apparent incongruence is that there is a gradual breakdown of the verbal code that is substantially less marked for numbers than for non-number words by virtue of the fact that numbers retain meaningful magnitude referents. This partial preservation of number words might be analogous to the better comprehension that SD patients show for words and objects with a day-to-day significance in their personal lives (Snowden, Griffiths, \& Neary, 1994; Snowden, Griffiths, \& Neary, 1996a): semantics for these items is thought to be bolstered by the patients' relatively intact episodic memory. We similarly propose that the intact quantity representations in the inferior parietal lobe may ame- liorate the effects of domain-general lexical-semantic loss for number words to some extent; ultimately however, we might expect SD patients' understanding of number words to become impaired.

There is some suggestion of verbal number deficits both for multi-digit items and at a later stage of the condition, in line with this hypothesis. SD patients show poorer comprehension and ISR for low frequency multi-digit number words like 'million' and 'thirteen' relative to single-digit number words like 'three' and 'seven' (Jefferies et al., 2004b), consistent with the greater vulnerability of low frequency words and concepts in this condition (Funnell, 1995). Single-digit number words are also less phonologically/morphologically complex, acquired at a younger age, and refer to concepts that are easy to manipulate; factors which may contribute to their relative preservation. Secondly, a late-stage patient, FM, was impaired at producing and comprehending even the singledigit number words $1-9$, and started to make phonological errors in digit span like those that occur for poorly comprehended non-number words (Knott, 1998). In the present study, we replicated and extended these findings for a second patient with late-stage SD.

We examined the evolution of number processing in patient MK. Her deficits remained uncommonly pure in the sense that she did not show the behavioural/personality changes that typically accompany fronto-temporal dementia; as a result, she remained cooperative to testing even when her performance on tests of semantic memory had been at floor for several years, providing a rare opportunity to examine the eventual fate of number processing in SD. The advantage of this longitudinal approach is that relatively subtle numerical difficulties purportedly associated with impairment of the verbal number code (see Lemer et al., 2003) should become more marked as the disease evolves. We examined performance in a range of tasks that would be expected to reveal deficits if the verbal code for numbers degrades in SD: namely transcoding between Arabic numerals and English number words, reading aloud number words, production and comprehension of number words in relation to arrays of dots and digit span. Knowledge of the magnitude and sequence of numbers was assessed with comparison and card-ordering tasks that utilised dot pictures, Arabic numerals and English number words. Success at these number knowledge tasks might be expected to follow the sequence dot pictures > Arabic numerals > English number words, if the verbal but not the parietal-magnitude representation of numbers degrades in this condition. We studied the patient's competence in everyday tasks involving number: specifically, telling the time and using money: these domains may be largely intact in SD if they draw on a relatively preserved understanding of numerical magnitude. We also looked at comprehension of non-number words that nevertheless had a dimensional meaning-for example, big/small. Although these words are likely to degrade in SD, they may, like number words, derive some protection from the unimpaired parietal magnitude system. 


\section{Case description}

This research was approved by the local health authority ethics committee and informed consent was obtained. Patient MK, a right-handed British woman, had previously been employed in clerical work and left school aged 17. In 2000, at the age of 66 years, she presented with a worsening difficulty in word finding and comprehension that her family had first noticed 1 or 2 years earlier. No other impairments were reported at this stage. An MRI scan showed marked temporal lobe atrophy that was strongly lateralised to the left side, with relative preservation of more medial temporal lobe structures including the hippocampus (see Fig. 1). Other cortical regions were less affected, including the interparietal sulcus thought to represent numerical magnitude, occipital-temporal cortex purportedly critical for the visual number code and the perisylvian language areas thought to subserve the verbal number code (see Dehaene \& Cohen, 1995).

Neuropsychological assessment at 2 years post onset (2001) revealed a relatively specific impairment of semantic memory that was already very severe (see Table 1). MK performed at or near chance on both verbal and visual semantic tests (e.g., the Pyramids and Palm Trees Test; Howard \& Patterson, 1992). She was extremely anomic in picture nam- ing and word fluency tasks. The majority of her naming errors were "don't know" responses (76\%). She also made a small number of semantic errors (e.g., cow $\rightarrow$ "dog"), superordinate responses (e.g., owl $\rightarrow$ "bird") and descriptions (e.g., comb $\rightarrow$ "it's for your hair"). In contrast to her profound semantic impairments, she remained well oriented in time and place, although she was impaired on the Mini Mental State Examination (Folstein, Folstein, \& McHugh, 1975) because of her severe comprehension difficulties. Her speech was fluent, syntactically well formed and free from both phonological errors and articulatory problems. She had good autobiographical memory and episodic memory for recent events. Her verbal STM was normal as assessed by forwards and backwards digit span and she also performed well on the Corsi spatial span task (taken from Wechsler, 1997). In common with other SD patients, her ISR was better for words that she still understood relatively well, compared with more semantically degraded words (Jefferies et al., 2004a). She showed a pattern of surface dyslexia in single word reading (e.g., she made regularisation errors, pronouncing PINT to rhyme with MINT) and surface dysgraphia in spelling tasks (e.g., writing TOMB as TOOM). She was able to copy the Rey complex figure accurately (taken from Lezak, 1976). She performed well on a number of tests of visuospatial pro-
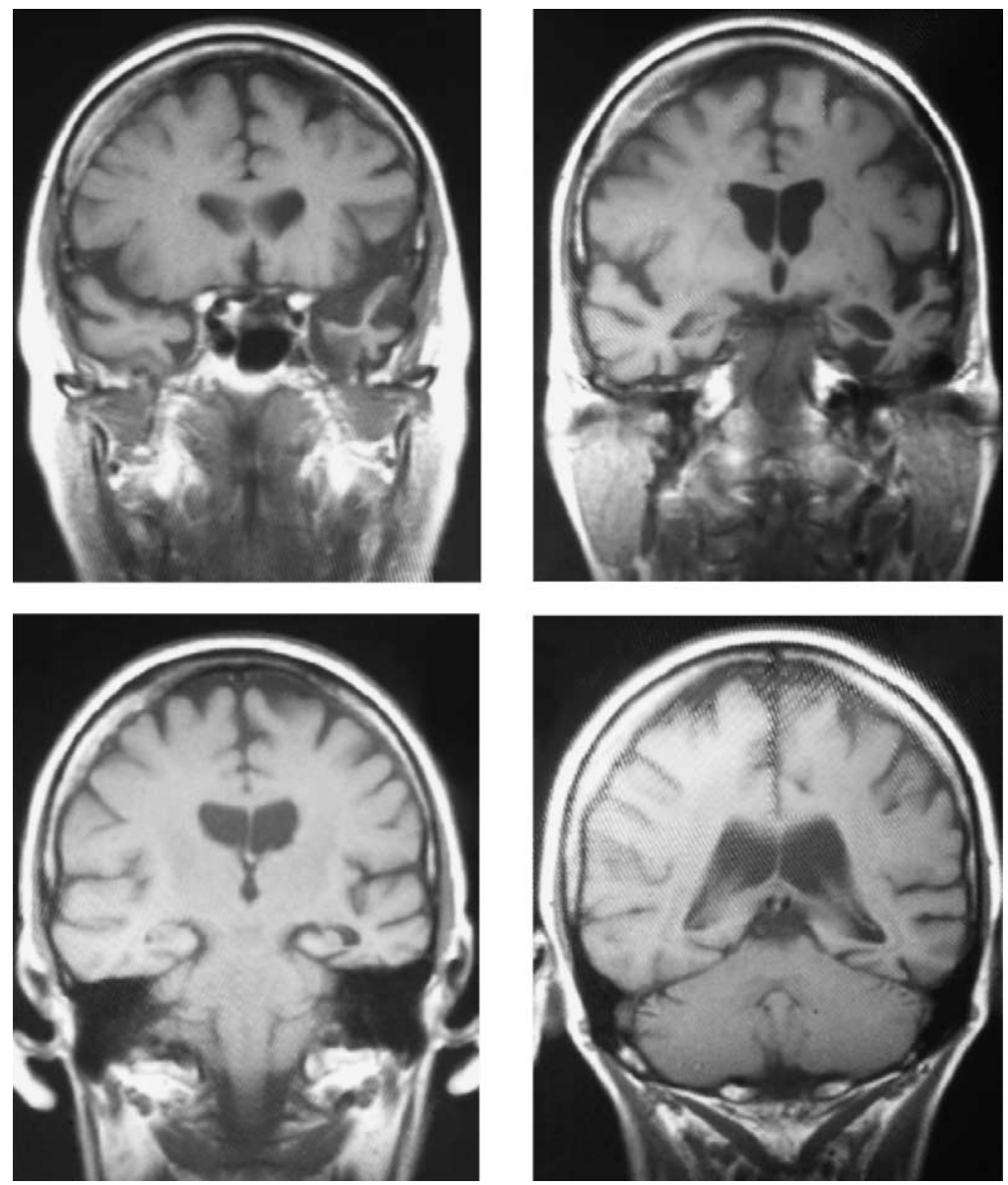

Fig. 1. MRI scan of patient MK from 2001: the four coronal sections show marked atrophy of the anterior infero-temporal cortex. 
Table 1

Background neuropsychological data

\begin{tabular}{|c|c|c|c|c|c|c|c|}
\hline \multirow[t]{2}{*}{ Test } & \multirow[t]{2}{*}{ Maximum score } & \multirow[t]{2}{*}{ MK } & & & & \multicolumn{2}{|c|}{ Normal controls } \\
\hline & & & & & & $\bar{M}$ & S.D. \\
\hline Years post onset & & 2 & 3 & 3.5 & 4 & & \\
\hline Age & & 66 & 67 & 68 & 68 & & \\
\hline MMSE $^{\mathrm{a}}$ & 30 & $21^{*}$ & $8^{*}$ & $4^{*}$ & - & $>24^{\mathrm{b}}$ & - \\
\hline Naming & 64 & $2^{*}$ & $3^{*}$ & - & - & $62.3^{\mathrm{c}}$ & $1.6^{\mathrm{c}}$ \\
\hline Word-picture matching ${ }^{\mathrm{d}}$ & 64 & $11^{*}$ & $11^{*}$ & $10^{*}$ & - & $63.7^{\mathrm{c}}$ & $0.5^{\mathrm{c}}$ \\
\hline PPT: pictures ${ }^{\mathrm{e}}$ & Chance $26 / 52$ & $33^{*}$ & $31^{*}$ & - & - & $51.1^{\mathrm{c}}$ & $1.1^{\mathrm{c}}$ \\
\hline PPT: words ${ }^{\mathrm{e}}$ & Chance $26 / 52$ & $26^{*}$ & $31^{*}$ & - & - & $51.2^{\mathrm{c}}$ & $1.4^{\mathrm{c}}$ \\
\hline Category fluency (eight categories) & - & $1^{*}$ & $1^{*}$ & - & - & $113.9^{\mathrm{k}}$ & $12.3^{\mathrm{k}}$ \\
\hline Letter fluency $(\mathrm{F}, \mathrm{A}, \mathrm{S})$ & - & $2^{*}$ & $7^{*}$ & $7^{*}$ & - & $44.2^{\mathrm{c}}$ & $11.2^{\mathrm{c}}$ \\
\hline Coloured progressive Matrices ${ }^{\mathrm{f}}$ & 36 & $22^{*}$ & $15^{*}$ & - & $14^{*}$ & - & - \\
\hline Digit span: forwards ${ }^{\mathrm{g}}$ & - & 5 & 5 & 5 & $4^{*}$ & $6.8^{\mathrm{c}}$ & $0.9^{c}$ \\
\hline Digit span: backwards ${ }^{g}$ & - & 4 & 3 & nu & - & $4.7^{\mathrm{c}}$ & $1.2^{\mathrm{c}}$ \\
\hline Spatial span: forwards ${ }^{\mathrm{h}}$ & - & - & 5 & 6 & 5 & $5-6^{\mathrm{i}}$ & - \\
\hline Spatial span: backwards ${ }^{\mathrm{h}}$ & - & - & 4 & nu & - & & - \\
\hline Rey figure immediate copy ${ }^{j}$ & 36 & 30 & 35 & 31 & 31 & $34.0^{\mathrm{k}}$ & $2.9^{\mathrm{k}}$ \\
\hline VOSP: screening test & 20 & 17 & 20 & 20 & - & & \\
\hline VOSP: incomplete letters ${ }^{1}$ & 20 & $10^{*}$ & $4^{*}$ & - & - & $19.2^{\mathrm{c}}$ & $0.8^{\mathrm{c}}$ \\
\hline VOSP: dot counting ${ }^{1}$ & 10 & 10 & 10 & 10 & - & $9.9^{c}$ & $0.3^{\mathrm{c}}$ \\
\hline VOSP: position discrimination ${ }^{1}$ & 20 & $17^{*}$ & 18 & nu & - & $19.8^{\mathrm{c}}$ & $0.6^{\mathrm{c}}$ \\
\hline VOSP: number location & 10 & $6^{*}$ & 8 & 8 & - & & \\
\hline VOSP: cube analysis ${ }^{1}$ & 10 & 6 & $2^{*}$ & - & - & $9.7^{\mathrm{c}}$ & $2.5^{\mathrm{c}}$ \\
\hline
\end{tabular}

nu Denotes task not understood.

Span scores: maximum length repeated correctly.

* Denotes abnormal performance.

${ }^{a}$ Mini-mental state examination (Folstein et al., 1975).

${ }^{\mathrm{b}}$ Cutoff for normal performance.

c Control data from Bozeat et al. (2002).

d Word-picture matching task included nine semantically related distracters.

e Pyramids and Palm Trees Test (Howard \& Patterson, 1992).

${ }^{\mathrm{f}}$ Raven's coloured progressive matrices (Raven, 1962).

g Weschler Memory Scale-Revised (Wechsler, 1987).

h Weschler Memory Scale-III (Wechsler, 1997).

${ }^{i}$ Normal range for age matched participants.

j Rey figure taken from Lezak (1976).

${ }^{k}$ Control data from Hodges and Patterson (1995).

${ }^{1}$ Visual object and space perceptual battery (Warrington \& James, 1991).

cessing (taken from the VOSP, Warrington \& James, 1991), although she showed some weakness on several other tests from this battery (tests of position discrimination and number location). She was also impaired on the Progressive Matrices Test of non-verbal reasoning (Raven, 1962). However, she did not show signs of disinhibition or other behavioural abnormalities and her family reported that her personality was unchanged. It was noticed that she frequently used numbers in spontaneous speech, for example, to describe the ages of her grandchildren. She used a calendar to record appointments and could tell the time.

Over the next few years it seems likely that MK's semantic impairment became even more severe, although this is not well documented in Table 1 because her performance was already at floor when she was first tested. At 4 years post onset (2003), her spontaneous speech was dominated by stereotyped phrases and her expressive vocabulary was severely limited. She began to make a number of phonological errors in naming, spontaneous speech and immediate repetition (Jefferies, Patterson, Bateman, Jones, \& Lam- bon Ralph, submitted-b). In addition, she had difficulties identifying individual letters, which led to a sharp increase in the number of words given implausible pronunciations and a decline in reading words with regular spellings. Her spelling deteriorated sharply over the same period. It should be noted that MK's performance fell on some tests of visualspatial skills and non-verbal reasoning, but these difficulties may have reflected failures to understand the instructions and possibly also semantic contributions to object recognition/segmentation (see Rogers, Lambon Ralph, Hodges, \& Patterson, 2003) - for example, she became severely impaired on the cube analysis subtest of the VOSP because she counted individual lines rather than the cubes they depicted. Her copy of the Rey figure remained good.

MK was not globally impaired even at the last examination (2004). Her speech remained fluent and largely syntactically intact. It appeared that her day-to-day memory was still relatively good, although she did not recognise the examiner when a period of several months had elapsed between visits. She could still find her way around very familiar places 
close to her home. MK remained free from the behavioural characteristics of fronto-temporal dementia and she continued to cooperate during testing, although formal testing was often impossible because she understood very little of what was said to her. Although she did not become socially withdrawn, her verbal utterances became less frequent towards the end of the study.

\section{Experimental investigations of the verbal number code}

\subsection{Reading and writing Arabic digits}

If the comprehension of English number words and/or recognition of Arabic numerals eventually declines in SD in parallel with the understanding of non-numerical words and pictures, MK should show deficits in transcoding between these stimuli.

\subsubsection{Method}

MK was asked to read aloud Arabic numbers printed in a large font (Arabic to verbal transcoding) and write Arabic numbers to dictation (verbal to Arabic transcoding). The stimuli in both tests were the single-digit numbers $1-9$, the two-digit numbers 10-19, the decades 20-90 and two larger numbers, 100 and 1000. The test was presented four times; at 2, 3, 4 and 5 years post onset. On the first presentation, the items 15, 20, 30, 40, 50, 100 and 1000 were not included.

\subsubsection{Results}

MK's performance on this task gradually deteriorated (see Table 2). When she was first tested at 2 years post onset, she was able to read and write Arabic numbers perfectly. A year later, her reading of Arabic numbers was still accurate but she made frequent errors in writing Arabic numbers to dictation. She wrote the digits 1-9 without error but showed poor performance for multi-digit numbers. Many of her errors (42\%) were of the form "nineteen" $\rightarrow 90$ or "seventy" $\rightarrow 17$. A further third of her errors were code intrusion errors, in which letters were produced instead of Arabic numbers; she wrote 11 as "L ...", 16 as " $1 \mathrm{~N}$ " and 70 as " $\mathrm{t}$ ". At 4 years post onset, she was impaired at writing single-digit numbers; almost all of her erroneous responses were single letters, frequently the first letter of the number word she was attempting to write (e.g., $5 \rightarrow$ F). At this stage, her reading of multi-digit Arabic numbers was also poor although she was able to read single digit numbers correctly. She read multi-digit numbers as single digit numbers (e.g., $80 \rightarrow$ "eight"; $17 \rightarrow$ "seven") or produced each component digit separately (e.g., $15 \rightarrow$ "one five"; $20 \rightarrow$ "two oh"). Her reading of Arabic numbers had worsened still further by 5 years post onset.

At 4 years post onset, MK's newly emerged difficulty in transcoding from multi-digit Arabic numbers to spoken English number words was explored more fully. She was asked to read aloud 10 two-digit numbers, 20 three-digit numbers and 10 four-digit numbers. Her accuracy was $0 \%$. She read each component digit separately in every trial (e.g., 120 $\rightarrow$ "one two oh", rather than "one hundred and twenty"). She very occasionally produced the wrong digit (e.g., $317 \rightarrow$ "five one seven") or read a numeral as a letter (e.g., $8 \rightarrow$ "X"). Several more of these number $\rightarrow$ letter errors were noted when MK was asked to read the Arabic numbers 1-9 twice, mixed with the capital letters A-Z (e.g., $5 \rightarrow$ "S", $1 \rightarrow$ "I"). In addition, she read some of the letters as numbers (e.g., G $\rightarrow$ "6", I $\rightarrow$ "1"), confused the letters for each other (e.g., Y $\rightarrow$ "J", $Z \rightarrow$ "X"), and made some responses that were neither letters nor numbers (e.g., $\mathrm{W} \rightarrow$ "wut", $8 \rightarrow$ "age"). Her overall accuracy was $78 \%$ for single digit numbers and $73 \%$ for letters.

\subsubsection{Discussion}

At the beginning of the study, MK's transcoding abilities were intact-this is consistent with other SD patients in the literature (Cappelletti et al., 2001; Diesfeldt, 1993). At a late-stage, however, MK showed marked impairment in both reading and writing Arabic numbers. Her errors did not fall within the standard lexical ("eight" $\rightarrow 4$ ) versus syntactic ("one thousand two hundred" $\rightarrow$ 1000200) dichotomy (Deloche \& Seron, 1982a; McCloskey, Sokol, \& Goodman, 1986). Instead she made frequent code intrusion errors in writing numerals to dictation (these can also occur in patients with Alzheimer's disease: Kessler \& Kalbe, 1996; Thioux, Ivanoiu, Turconi, \& Seron, 1999). In addition, MK showed a specific confusion of teens and decades (e.g., 17 and 70), in line with the hypothesis that the meanings of these words were partly degraded. According to some authors (Deloche \& Seron, 1982a, 1982b; Seron \& Deloche, 1983, 1984), verbal numbers are organised into lexical classes (units, teens, tens, etc.) and positions within classes (first, second, third, etc.): accordingly two specific types of error (failure to select lexical class and position within class) have been observed

Table 2

Reading and writing to dictation for Arabic numerals at 2, 3, 4 and 5 years post onset

\begin{tabular}{|c|c|c|c|c|c|c|c|c|c|}
\hline \multirow[t]{2}{*}{ Items } & \multirow[t]{2}{*}{$N$} & \multicolumn{4}{|c|}{ Reading Arabic numbers } & \multicolumn{4}{|c|}{ Writing Arabic numbers } \\
\hline & & 2 years & 3 years & 4 years & 5 years & 2 years & 3 years & 4 years & 5 years \\
\hline $1-9$ & 9 & 100 & 100 & 100 & 89 & 100 & 100 & 22 & NT \\
\hline $10-19$ & $10(9)$ & 100 & 100 & 50 & 11 & 100 & 40 & NT & NT \\
\hline $20-90$ & $8(4)$ & 100 & 100 & 13 & 0 & 100 & 38 & NT & NT \\
\hline 100,1000 & 2 & NT & 50 & 0 & 0 & NT & 50 & NT & NT \\
\hline
\end{tabular}

Figures denote percentage of items correct; $N$ : number of items (in parentheses, the number of items on the first presentation only); NT: not tested. 
in brain-damaged patients. MK's errors preserved position within a class but not the class itself: e.g., $17 \rightarrow 70,50 \rightarrow 15$. Finally, her tendency to read multi-digit numbers as a string of single digits may have reflected her difficulties in producing lower-frequency multi-digit number words.

\subsection{Naming and word-picture matching with dot pictures}

In order to further explore MK's production and comprehension of English number words, she was asked to name numbers that were represented pictorially as dots and perform word-picture matching with dot pictures.

\subsubsection{Method}

There were two tests; one comprised the numbers 1-10 and the other involved a set of 12 larger numbers, 10 of which were multi-digit numbers (the numbers 8-14, 16, 18, 19, 60 and 70: this is referred to as the "multi-digit" set in Table 3). Tens were depicted as clusters of 10 dots, and units were depicted as single dots. For example, the word 'thirteen' was shown as a cluster of 10 dots and three individual dots. MK was told that the clusters contained 10 dots. In naming, MK was asked to provide a name for each dot picture in turn. In word-picture matching, she was shown all the pictures together and asked to point to the one that represented a particular spoken number word. She was tested three times on the multi-digit numbers at 2, 3 and 4 years post onset. The digits 1-10 were examined three times, at 3, 4 and 5 years post onset. Word-picture matching was not tested at 4 years post onset because MK was unable to comprehend the test instructions. At 5 years post onset, comprehension of the task was facilitated by (1) presenting both written and spoken English number names and (2) preceding the task with a non-verbal version (see below).

\subsubsection{Results}

The results are shown in Table 3. When MK was first tested, her performance was virtually perfect. A year later, she was able to correctly name and select dot pictures representing the numbers $1-10$ but she showed much poorer performance with multi-digit numbers. She used an ineffi- cient 'counting up' strategy to produce multi-digit number words: e.g., for the item 60, she did not use the clusters of 10 dots to count through the decades, but instead attempted to count each dot separately. MK may have adopted this counting technique because of a difficulty in spontaneously producing lower frequency multi-digit number words. She occasionally miscounted, particularly around the decade boundaries (e.g., "39, 40, 31”), resulting in errors. At 4 years post onset, MK no longer produced any lower frequency multidigit number words in the naming task. She used the numbers $1-10$ to describe all of the pictures and showed considerable consistency in the word order that she adopted. Numbers from 11 to 19 were produced as "ten one", "ten two", "ten three", etc. The only exceptions were $16 \rightarrow$ "ten and three and three" and $18 \rightarrow$ "ten and four and four". The numbers 60 and 70 were produced as "six ten" and "seven ten". Therefore, her responses preserved the syntax of English numbers but reflected a substantial loss of vocabulary. At 5 years post onset, MK demonstrated that she was still capable of producing some multi-digit number words by counting up from one. Some of her errors resulted from failures to count correctly beyond 10 (e.g., $12 \rightarrow$ " $1,2,3,4,5,6$, $7,8,9,10,1,2$ ") and some were phonological in nature $(13 \rightarrow 1,2,3,4,5,6,7,8,9,10,11,12$, therefort $)$. She was also very impaired at matching the number words $1-10$ to arrays of dots. In this task, her incorrect selections did not suggest any approximate knowledge of the number words' magnitudes.

\subsubsection{Discussion}

This task revealed further weaknesses in MK's production and comprehension of number words, which were especially severe for multi-digit numbers.

\subsubsection{Transcoding between Arabic numbers and dot pictures}

At 5 years post onset, MK's ability to transcode between English number words and dot arrays (described above) was compared with her performance on the same tasks for Arabic numerals, to establish whether her difficulties were restricted to the verbal number code.

Table 3

Naming and word-picture matching with dot pictures

\begin{tabular}{|c|c|c|c|c|c|c|c|}
\hline & \multirow[t]{2}{*}{$N$} & \multicolumn{4}{|c|}{ Dot-English (naming)/English-dot (comprehension) } & \multirow{2}{*}{$\begin{array}{l}\text { Dot-Arabic (naming)/Arabic-dot } \\
\text { (comprehension) }\end{array}$} & \multirow{2}{*}{$\begin{array}{l}\text { Dot-dot } \\
5 \text { years }\end{array}$} \\
\hline & & 2 years & 3 years & 4 years & 5 years & & \\
\hline \multicolumn{8}{|l|}{ Naming } \\
\hline $1-10$ & 10 & NT & 100 & 90 & 90 & 0 & - \\
\hline Multi-digit $^{\mathrm{a}}$ & 12 & 100 & 83 & 17 & 58 & NT & - \\
\hline \multicolumn{8}{|l|}{ Comprehension } \\
\hline $1-10$ & 10 & NT & 100 & NT & 20 & 100 & $75^{\mathrm{b}}$ \\
\hline Multi-digit $^{\mathrm{a}}$ & 12 & 92 & 50 & NT & NT & NT & NT \\
\hline
\end{tabular}

Figures indicate the percentage of correct items at 2, 3, 4 and 5 years post onset; NT: not tested.

a This set comprised predominately multi-digit numbers (see text).

b $N=8$; only the numbers $1-8$ were tested. 


\subsubsection{Method and results}

MK was asked to write Arabic numerals for dot arrays representing the numbers $1-6$. The dot pictures were similar to those described above and were presented in a random order. She was shown several examples to facilitate her comprehension of the task. She scored 0/6 although she counted the dots aloud correctly on every trial. Her errors were $5 \rightarrow$ " 1 ", $1 \rightarrow$ "one", $6 \rightarrow$ "126" (corresponding to her verbal counting) and three omissions.

She was also tested on her ability to match cards representing the numbers 1-10 in non-symbolic, Arabic and English formats to an array of dot pictures. The task construction was exactly as for the word-dot matching task described above. In the first version of the task, MK matched non-symbolic numbers to dot arrays (e.g., three stars to three dots). The tokens that represented the same numbers had different spatial arrangements: hence, the arrays to be matched were superficially different. MK then performed the task with Arabic numerals and finally with written English words that were read aloud for her (these data are included in Table 3 and discussed above). The dot pictures that constituted the 10 targets/distracters were rearranged each time to limit the involvement of episodic memory.

As noted above, MK's performance with English number words was poor $(20 \%)$. Her matching of different dot arrays was also impaired (75\%). She performed this task very slowly for larger numbers and arduously counted and re-counted the tokens to find a match. Consequently, the items 9 and 10 were not tested. In contrast to her difficulty with English number words, she was fast and accurate at matching Arabic numbers to dot pictures $(100 \%)$.

\subsubsection{Discussion}

MK showed poorer production of Arabic numerals than spoken number words in response to dot arrays. Nevertheless, she was able to match Arabic numbers to arrays of dots, even when she could no longer perform this task for number words (see Table 3). MK's poor production of Arabic numbers in an entirely non-verbal task might suggest that, in line with her multimodal semantic impairment, her knowledge of both English and Arabic numbers was degrading.

\subsection{Reading English number words}

SD patients typically show better reading of number than non-number words (Cappelletti et al., 2002; Jefferies et al., 2004b), in line with the hypothesis that the correct pronunciation of these items receives more support from semantics (Butterworth et al., 2001). If the verbal code for numbers eventually degrades, however, MK might be expected to show a reduction in the size of this effect.

\subsubsection{Method}

MK was tested on the stimuli used by Cappelletti et al. (2002). She was asked to read 30 cardinal number words (the numbers from 1 to 20 , each tenth number-'thirty', 'forty', etc. and the words hundred, thousand and million). She was also asked to read 22 ordinal number words (first, second, etc. up to twentieth, and the items hundredth and thousandth), and 18 'ambiguous' number-related words that also had nonnumerical meanings (add, minus, share, etc.). These 70 number and number-related words were categorised as having regular and irregular spelling patterns and were compared with 70 non-number words matched on frequency, spelling regularity and length. The test was presented twice, at 2 and 4 years post onset.

\subsubsection{Results}

The number of items that MK read correctly fell substantially between the two test sessions at 2 and 4 years post onset $\left(\chi^{2}(1)=31.88, p<0.0001\right.$; see Table 4$)$. When first tested, she showed a marginally significant advantage for regular over irregular words $\left(\chi^{2}(1)=2.69\right.$, exact one-tailed $p=0.05)$. This regularity effect had disappeared by 4 years post onset $\left(\chi^{2}(1)<1\right)$. Collapsing across the two test sessions, MK showed better reading of cardinal number words than matched non-number words $\left(\chi^{2}(1)=2.91\right.$, exact onetailed $p<0.05)$, replicating the results of Cappelletti et al. (2002), although the effect was much weaker for our patient. The difference between cardinal number words and matched non-number words did not reach significance for either test session in isolation ( 2 years post onset: $\chi^{2}(1)<1$; 4 years post onset: $\chi^{2}(1)=1.67$, exact one-tailed $\left.p=0.1\right)$.

The percentage of phonemes produced correctly for each item provides a more sensitive measure of reading accuracy. In an analysis of variance (ANOVA), cardinal number words were read more accurately than matched nonnumber words $(F(1,56)=4.40, p<0.05)$. There was also a significant main effect of time since disease onset $(F(1$, $56)=14.10, p<0.001)$. The three-way interaction between word category (number versus non-number), regularity and test session reached significance $(F(1,56)=4.84, p<0.05)$. Planned comparisons revealed that for number words, irregular items were read significantly more poorly at a later stage of the condition $(t(10)=2.66, p<0.05)$ but regular number words did not show this decline $(t(18)=1.90, p=0.07)$. The drop in accuracy was presumably more severe for irregular number words because semantics plays a greater role in deriving the correct pronunciation of items with irregular spellings (Patterson \& Hodges, 1992). MK's reading of irregular non-number words was already severely impaired when she was first tested; consequently there was no significant decline in her reading of these items over time $(t(10)<1)$. She did show a significant decline in reading regular nonnumber words $(t(18)=3.09, p<0.01)$. To summarise: in common with other SD patients, MK's reading was more impaired for irregular than regular non-number words. Although number word reading was initially relatively well preserved, the regular $>$ irregular pattern was later mirrored for number words.

When MK was tested 2 years after the onset of her symptoms, she was able to read the number words $1-9$ with $100 \%$ 
Table 4

Reading accuracy for number and non-number words

\begin{tabular}{|c|c|c|c|c|}
\hline & \multicolumn{2}{|l|}{2 years post onset } & \multicolumn{2}{|l|}{4 years post onset } \\
\hline & Percentage items & Percentage of phonology: $M$ (S.D.) & Percentage items & Percentage of phonology: $M$ (S.D.) \\
\hline \multicolumn{5}{|c|}{ Cardinal number words $(N=30)$} \\
\hline Regular $(N=19)$ & 84 & $97(7)$ & 53 & $90(14)$ \\
\hline Irregular $(N=11)$ & 91 & $97(10)$ & 55 & $82(24)$ \\
\hline \multicolumn{5}{|c|}{ Ordinal number words $(N=22)$} \\
\hline Regular $(N=13)$ & 77 & $97(6)$ & 46 & $87(16)$ \\
\hline Irregular $(N=9)$ & 67 & $92(12)$ & 22 & $67(32)$ \\
\hline \multicolumn{5}{|c|}{ Cardinal + ordinal number words $(N=52)$} \\
\hline Regular & 81 & 97 & 50 & 89 \\
\hline Irregular & 80 & 95 & 40 & 75 \\
\hline \multicolumn{5}{|c|}{ Ambiguous number words $(N=18)$} \\
\hline Regular $(N=14)$ & 57 & $91(18)$ & 14 & $75(18)$ \\
\hline Irregular $(N=4)$ & 50 & $82(17)$ & 25 & $74(9)$ \\
\hline \multicolumn{5}{|c|}{ Non-number words matched to cardinal numbers $(N=30)$} \\
\hline Regular $(N=19)$ & 79 & $93(14)$ & 32 & $75(24)$ \\
\hline Irregular $(N=11)$ & 64 & $84(25)$ & 45 & $82(17)$ \\
\hline \multicolumn{5}{|c|}{ Non-number words matched to ordinal + ambiguous words $(N=30)$} \\
\hline Regular $(N=19)$ & 70 & $93(13)$ & 37 & $80(19)$ \\
\hline Irregular $(N=11)$ & 46 & $90(11)$ & 31 & $78(23)$ \\
\hline \multicolumn{5}{|c|}{ All non-number words $(N=70)$} \\
\hline Regular $(N=46)$ & 74 & $93(13)$ & 33 & $78(21)$ \\
\hline Irregular $(n=24)$ & 54 & $86(19)$ & 25 & $80(20)$ \\
\hline
\end{tabular}

accuracy (both as ordinals and as cardinals), but she erred to a greater extent on the numbers $10-19$ (80\% correct for cardinals, 50\% for ordinals) and the decades $20-90$ (75\% of cardinals correct). This advantage for single-digit numbers was significant $\left(\chi^{2}(1)=5.30, p<0.05\right)$. Four years post onset, the advantage for the numbers 1-9 was no longer apparent $\left(\chi^{2}(1)<1\right)$; accuracy had dropped to 55 and $44 \%$ for cardinals and ordinals, respectively (versus 50 and $30 \%$ for cardinal/ordinal numbers from 10 to 15 , and $63 \%$ for cardinals from 20 to 90 ).

MK's reading errors were a mixture of legitimate alternative reading of components (LARC errors, Patterson, Suzuki, Wydell, \& Sasanuma, 1995) and implausible responses. LARC errors were plausible in the sense that each component was pronounced in a way that would be appropriate in a different word; they occurred for both regular words (PATIO $\rightarrow$ "paytio"; FIFTEEN $\rightarrow$ "fife-teen") and irregular words (BASIC $\rightarrow$ "bassic"; NINTH to rhyme with "plinth"). Implausible responses frequently resulted from letter misidentifications (which may occur increasingly in SD as the condition worsens: Graham, Patterson, \& Hodges, 2001); examples are COTTON $\rightarrow$ "totton" and FORTY $\rightarrow$ "porty". A few errors contained both plausible and implausible elements, as in BRAND $\rightarrow$ "grant". Number and non-number words did not differ in terms of the balance of LARC to non-LARC errors. However, the proportion of errors that were non-LARC/mixed was marginally higher for ordinal as opposed to cardinal number words (39\% of 18 errors and $11 \%$ of 19 errors, respectively; Fisher's exact test, $p=0.06$ ). Some of these implausible pronunciations of or- dinal words included the production of inappropriate number words (SIXTEENTH $\rightarrow$ "six-tenth"; THIRTEENTH $\rightarrow$ "thir-tenth").

\subsubsection{Discussion}

At a late-stage of the condition, patient MK developed an impairment of number word reading, especially for items with irregular spellings, which mirrored her earlier difficulties with non-number words.

\subsection{Digit span}

Digit span appears to be unimpaired in the early to mid stages of SD, but patients typically show a severe deficit in word span relative to controls (Jefferies et al., 2004b; Warrington, 1975). Moreover, digit recall is characterised by a relatively normal pattern of omission, order and intrusion errors, whereas phonological errors occur at an elevated rate in the recall of non-number words. This ISR advantage for digits remains substantial even when frequency, imageability, word length, set size and size of semantic category are matched across the two types of material (Jefferies et al., 2004b). The ISR differences between number and non-number words are reminiscent of those observed for relatively well-known versus semantically degraded words (Jefferies et al., 2004a; Knott et al., 1997; Patterson et al., 1994), suggesting that a comprehension difference might underpin the better ISR for numbers. Indeed, there is an association between recall and comprehension for number versus non-number words. Similarly, within the number domain, SD patients show bet- 
Table 5

Accuracy of immediate serial digit recall

\begin{tabular}{|c|c|c|c|c|c|c|}
\hline \multirow[t]{2}{*}{ List length } & \multicolumn{4}{|l|}{ MK } & \multicolumn{2}{|l|}{ Controls } \\
\hline & 2 years & 3 years & 3.5 years & 4 years & Mean & Minimum \\
\hline 5 & $94(9.7)$ & $72^{*}(21.5)$ & $76^{*}(15.8)$ & $41^{*}(20.1)$ & $95(3.6)$ & 86 \\
\hline 6 & $90(14.1)$ & $63^{*}(18.9)$ & $70(18.2)$ & $41^{*}(15.5)$ & $89(9.7)$ & 70 \\
\hline 7 & $79(18.1)$ & $47^{*}(9.6)$ & $61^{*}(11.8)$ & NT & 82 (11.6) & 63 \\
\hline
\end{tabular}

Figures for MK indicate the average percentage of items recalled per list at 2, 3, 3.5 and 4 years post onset. For the control participants, a group average of this figure is indicated (S.D. in parentheses in both cases); NT: not tested.

* Denotes performance below normal range.

ter comprehension and recall of single-digit numbers versus multi-digit numbers, consistent with the view that digit span is intact in SD because patients retain a good understanding of the words 1-9 (Jefferies et al., 2004b). These findings concord with the view that that lexical and semantic representations make an important contribution to the maintenance of phonology over brief periods of time (Martin \& Saffran, 1997; Patterson et al., 1994).

Although digit span has been noted to remain relatively stable in the face of marked semantic decline (Knott et al., 2000), this task presumably draws heavily on the verbal number code and so, in line with Lemer et al.'s (2003) hypothesis, should not be immune to the effects of lexical-semantic degradation at a late stage of the condition. We expect to see a decline in MK's ISR for digits, as well as an emergence of phonological errors in this task, given her poor production and comprehension of these items. Similar findings have already been reported for one late-stage SD patient (Knott, 1998).

\subsubsection{Method}

Five, six and seven digit lists were presented auditorily at a rate of one item per second for immediate serial recall. There were 10 lists at each list length. MK was tested four times; at $2,3,3.5$, and 4 years post onset. The seven-item lists were not tested at 4 years post onset. Twelve healthy controls, matched to the patient for age and educational level, were also tested on these materials.

\subsubsection{Results}

Table 5 shows recall accuracy for MK and the controls. At 2 years post onset, MK's digit recall was within the nor- mal range on every list length (in sharp contrast to her recall of non-number words which she comprehended poorly; see Jefferies et al., 2004b). Over the next 2 years, her ISR for digits gradually declined and it was severely impaired by the end of the study. She showed a significant drop in recall accuracy from 2 to 3 years post onset $(t(29)=6.42, p<0.0001)$, no significant difference between the assessments at 3 and 3.5 years post onset $(t(29)=1.89, p=0.07)$ and a substantial decline from 3.5 to 4 years post onset $(t(19)=6.60$, $p<0.0001$ ).

Incorrect responses were categorised as omission, order, repetition, intrusion, phonological and unrelated errors. Omission errors were calculated by subtracting correct responses and other error types from the number of items presented. Responses were counted as an order error if the word produced was a target word occurring somewhere else in the sequence. Repetition errors were target words recalled more than once. Intrusion errors were digits not present in the target list. Phonological errors were divided into two categories; those sharing at least $50 \%$ of the phonemes from the target word (e.g., "five" $\rightarrow$ "fide", "four" $\rightarrow$ "ford"), and those containing fewer than $50 \%$ but at least one of the target phonemes (e.g., "seven" $\rightarrow$ "ferret", "five" $\rightarrow$ "eye"). A small number of unrelated errors did not fall into any of these categories.

When MK was first tested, she did not make any phonological errors in digit recall in line with the performance of controls (see Table 6). Her errors were omissions, order errors, repetitions and intrusions. The number of phonological errors increased steadily as her condition progressed. A greater number of her errors were phonological at 3 versus 2 years post onset $\left(\chi^{2}(1)=7.47\right.$,

Table 6

Errors in digit recall

\begin{tabular}{|c|c|c|c|c|c|c|}
\hline & \multicolumn{4}{|l|}{ MK } & \multicolumn{2}{|c|}{ Controls } \\
\hline & 2 years & 3 years & 3.5 years & 4 years & Mean & Maximum \\
\hline Phonological: >50\% shared & 0 & $19^{*}$ & $23^{*}$ & $35^{*}$ & 0 & 0 \\
\hline Phonological: at least one shared & 0 & $10^{*}$ & $4^{*}$ & $20^{*}$ & 0 & 0 \\
\hline Unrelated & 0 & 0 & $1^{*}$ & $5^{*}$ & 0 & 0 \\
\hline Omission & 40 & 12 & 12 & 28 & 20 & 58 \\
\hline Order & 32 & 21 & 18 & 5 & 45 & 100 \\
\hline Repetition & 8 & 23 & 25 & 5 & 19 & 100 \\
\hline Intrusion & 20 & 15 & 16 & 3 & 15 & 29 \\
\hline
\end{tabular}

Figures indicate the number of errors of each type as a percentage of the total number of errors at 2, 3, 3.5 and 4 years post onset.

* Denotes performance outside normal range. 
$p<0.01)$. She also made more phonological errors at 4 versus 3.5 years post onset $\left(\chi^{2}(1)=10.04, p<0.01\right)$. Nonphonological errors (e.g., order and repetition errors), in contrast, did not show any marked increase over the period of the study, in line with the view that lexical-semantic knowledge makes a particular contribution to phonological coherence in ISR (Jefferies, Frankish, \& Lambon Ralph, submitteda; Patterson et al., 1994). MK showed some decline in order and digit intrusion errors longitudinally, presumably because these errors require number words to be produced correctly.

\subsubsection{Discussion}

MK started to make phonological errors in digit span as her comprehension and production of verbal numbers declined. These were similar to those shown by patients with less severe SD in the recall of semantically degraded nonnumber words (Jefferies et al., 2004a; Knott et al., 1997; Patterson et al., 1994). SD patients make phonological errors in ISR for poorly comprehended words even in the absence of additional phonological problems (Jefferies et al., in press), suggesting that semantics provides an important source of constraint on phonological processing in the normal system. Input from semantics is thought to contribute to the maintenance of phonological activation (Martin \& Saffran, 1997) and may encourage the phonemes of familiar words to emerge together as a coherent item (Jefferies et al., submitted-a; Patterson et al., 1994). Patients with less severe SD also make phonological errors in their recall of multi-digit number words, and they show some comprehension problems for these items (although comprehension/recall of these numbers is still substantially better than for matched non-number words; Jefferies et al., 2004b).

MK's declining digit span may have reflected a broadening of these difficulties. The appearance of phonological errors was associated with the emergence of deficits in the naming of single-digit numbers (although these were milder than those for multi-digit numbers), suggesting that MK's digit span impairment may have resulted from a reduction in the normal lexical-semantic contribution to verbal STM. This explanation is consistent with the viewpoint that the verbal code for numbers degrades in late-stage SD, even though number words initially derive some protection from global lexical-semantic decline by virtue of their meaningful referents in the parietal lobe magnitude system. It is also interesting to note that MK began to show similar phonological errors in ISR for letters ( $\rightarrow \rightarrow$ "chew"), in line with her poor understanding and production of these items (Jefferies et al., submitted-b). Although MK's phonological errors in digit span co-occurred with the emergence of phonological errors in her spontaneous speech, naming and immediate single word repetition, her phonological errors in all of these tasks may have reflected the impact of severe semantic degradation on phonological processing rather than the emergence of an independent phonological deficit (Jefferies et al., submittedb; see General Discussion).

\section{Knowledge of number magnitude and sequence}

MK's performance was examined on two tasks thought to tap possibly dissociable aspects of number knowledge: magnitude comparison and number ordering (Delazer \& Butterworth, 1997). In each case, MK's performance was compared for non-symbolic quantities, Arabic numerals and English number words.

\subsection{Magnitude comparison}

Magnitude comparison with Arabic numbers should be relatively intact in SD because this task is thought to draw heavily on representations of numerical magnitude while being relatively independent of linguistic representations (but see Noel \& Seron, 1997). If the stimuli are presented as English number words however, a greater impairment might be anticipated.

\subsubsection{Method}

MK was examined three times on a magnitude comparison task involving Arabic numbers, at 2, 3 and 4 years post onset (she was unable to understand the task at 5 years post onset). There were 20 questions. Six involved comparisons between single-digit numbers (e.g., 9 and 2), two involved one versus two-digit numbers (e.g., 7 and 13) and eight involved comparisons between two-digit numbers (e.g., 10 and 16). The numerical distance between the numbers to be compared ranged from 2 to 7 for both the larger two-digit numbers and the smaller single-digit numbers. A further two stimuli examined two versus three-digit numbers (e.g., 105 and 89) and two involved comparisons between three-digit numbers (e.g., 948 and 199). The difficulty of these comparisons is likely to have varied with both the numerical size of the numbers to be compared and the distance between them (Dehaene, 1989; Dehaene, Dupoux, \& Mehler, 1990; Moyer \& Landauer, 1967). The correct response was positioned on the left- and right-hand side of the page in an equal number of trials. MK was under no pressure to respond quickly and reaction times were not recorded.

MK was also tested twice (at 3 and 4 years post onset) on the eight pairs of '-teen' and '-ty' words from " 12 and 20 " to " 19 and 90 ", in order to determine if she showed any tendency to confuse these numbers. Numerical distance (expressed as a proportion of the size of the numbers to be compared) was larger for these '-teen' versus '-ty' items than for the comparisons between two-digit numbers described above $(M=1.03$, S.D. $=0.27$ and $M=0.24$, S.D. $=0.15$, respectively; $t(14)=7.13, p<.001)$, suggesting that any inherent difference in difficulty between the two sets of items would have favoured the '-teen' versus '-ty' items.

These comparisons were presented as Arabic numerals (e.g., 9 and 2) and number words (e.g., NINE and TWO) at 4 years post onset, so that MK's performance could be compared across the number formats. The four trials involving three-digit numbers were not presented as number words. 
Table 7

Magnitude comparison performance

\begin{tabular}{lllccc}
\hline & $N$ & \multicolumn{2}{l}{ Arabic } & & English \\
\cline { 2 - 4 } & & 2 years & 3 years & 4 years & 4 years \\
\hline $\begin{array}{l}\text { One to two digit } \\
\quad \text { comparison }\end{array}$ & 16 & 100 & 93.8 & 93.8 & 68.8 \\
$\begin{array}{l}\text { Three digit comparison } \\
\text {-Teen and -ty: e.g., 16 }\end{array}$ & 4 & 100 & 100 & 100 & NT \\
$\quad$ and 60 & 8 & NT & 75.0 & 100 & 12.5 \\
Total & & 100 & 89.3 & 96.4 & 50.0 \\
\hline
\end{tabular}

NT: not tested; figures indicate the percentage of correct trials at 2, 3 and 4 years post onset.

The Arabic numbers were presented before the number words in a separate block. The experimenter read aloud the number words, but not the Arabic numerals, because MK's number word reading was poor at this late stage, whereas her responses to Arabic numerals were fast and accurate. The nature of the task was illustrated with large and small circles. MK was able to select the larger of two circles without difficulty (3/3 trials correct).

\subsubsection{Results}

The results are shown in Table 7. MK's performance with Arabic numbers was errorless at 2 years post onset, and remained good when this test was repeated at 3 and 4 years post onset. Accuracy was reduced when the numbers were presented as English words rather than Arabic numerals at 4 years post onset (McNemar Test: $p<0.01)$, consistent with the suggestion that the verbal number code is specifically impaired in this condition. For these items, MK did not show better performance when numerical distance (expressed as a proportion of size) was large: mean distance/size for correct responses $=0.43$, S.D. $=0.14$; mean for incorrect responses $=0.91$, S.D. $=0.31$. MK did show a specific difficulty with "-teen" versus “-ty" trials (e.g., "16 and 60") — - her performance was significantly worse for this subset of trials $\left(\chi^{2}(1)=10.55, p<0.001\right)$. She did not show this impairment when tested with Arabic numerals, suggesting that she may have had a relatively intact understanding of the 'syntax' of numbers; i.e., she understood the importance of the position of the ' 6 ' in the numbers ' 16 ' and ' 60 '.

\subsubsection{Discussion}

MK's performance on the Arabic number comparison task remained remarkably intact especially given that, at a late stage of the condition, she could no longer reliably produce the names of multi-digit numbers (e.g., she read 20 as "two oh"). Number comparison in normal participants is thought to draw heavily on a non-verbal analogue representation of magnitude (Dehaene, 1989; Dehaene et al., 1990; Moyer \& Landauer, 1967; but see Noel \& Seron, 1997), suggesting that this code may be relatively preserved in late-stage SD. In contrast, MK showed a substantial impairment on the comparison task when it involved English number words, especially for the "-teen" versus "-ty" trials, consistent with the view that the verbal number code is specifically impaired in this condition.

MK's poorer performance with English number words relative to Arabic numerals might have reflected her predominately left-sided atrophy. In a semantic system distributed across both cerebral hemispheres, in which the left hemisphere has much stronger connections to phonology, leftsided atrophy is expected to cause greater problems with verbal than visual-based tasks (Lambon Ralph et al., 2001). Similarly, in the number domain, although both hemispheres can access the magnitude of Arabic numbers, the left hemisphere is thought to play a unique role in reading aloud number words (Cohen \& Dehaene, 1995a, 1996).

MK was able to access the magnitude of Arabic numbers but not number words, consistent with the suggestion that there are direct connections between Arabic numerals and their associated magnitudes that are not verbally mediated. These links are revealed by patients' abilities to process the magnitude meaning of Arabic numbers that they cannot read (Cipolotti \& Butterworth, 1995; Cohen \& Dehaene, 1995b; Delazer \& Bartha, 2001; Whalen, McCloskey, Lindemann, $\&$ Bouton, 2002) and by the automatic access to magnitude from Arabic numerals shown by normal participants (e.g., Dehaene, Bossini, \& Giraux, 1993; Fischer, Castel, Dodd, \& Pratt, 2003).

\subsection{Knowledge of number sequence}

It was noted that MK made occasional errors while counting dots aloud (see above). In order to formally assess her knowledge of number sequence, her ability to arrange numbers in order was compared for non-symbolic arrays of dots, Arabic numerals and number words. Accuracy was expected to be highest for non-symbolic representations of magnitude, poorer for Arabic numerals and poorer still for number words, in line with the magnitude comparison findings above.

\subsubsection{Method}

MK was given nine cards, representing the numbers 1-9 and was asked to sequence them. To facilitate understanding, the experimenter selected the first card and said "One-what comes next? One and then ...". MK performed the task first with dots, then with Arabic numerals and finally with English number words. MK was tested at 4 and 5 years post onset. She had previously been asked to arrange the English number words in order at 2 years post onset. At 4 years post onset, MK was able to count the dots and read the Arabic and English numbers aloud without error throughout the test- the experimenter did not provide spoken number names. At 5 years post onset, the experimenter read the number words as MK looked at each card.

\subsubsection{Results}

At 2 years post onset, MK arranged the English number words from 1 to 9 in sequence without error (although, as reported in a previous study, her performance was less good 
for multi-digit numbers; Jefferies et al., 2004b). In contrast, at 4 years post onset, she produced the sequence " $1,2,8,9$, $5,4,3,7,6$ "- -even though she read all of the number words correctly and performed flawlessly with dot arrays and Arabic numbers. These findings were replicated at 5 years post onset: she produced the sequence $1,2,8,9,5,6,7,4,3$ for number words and was again errorless for the dot arrays and Arabic numbers. Therefore, at a late-stage of the condition, MK showed a specific difficulty in ordering English number words while demonstrating good knowledge of number sequence for other formats.

MK's ability to order Arabic numerals was explored more fully at 5 years post onset. She performed faultlessly on a further five sets of nine numbers: (1) the units from 11 to 19 ; (2) the decades from 10 to 90; (3) a mixture of single unit, teens and decades numbers; (4) two-digit numbers; and (5) three-digit numbers from 100 to 500 .

\subsubsection{Discussion}

MK was able to sequence dot arrays and Arabic numbers but not number words.

\section{Calculation}

A dissociation between intact addition/subtraction contrasting with poorer multiplication/division performance has been described for several SD patients (Cappelletti et al., 2001; Jefferies et al., 2004b; Lemer et al., 2003) in line with the view that multiplication often involves the retrieval of arithmetic facts stored in a verbal code, whereas subtraction relies more heavily on magnitude representations (e.g., Dehaene \& Cohen, 1995). Impairments of subtraction, but not multiplication, are associated with parietal lobe damage and dysfunction of the quantity processing system (Dehaene $\&$ Cohen, 1997), and functional imaging studies reveal that subtraction tasks produce parietal activation in normal subjects (Chochon, Cohen, Van de Moortele, \& Dehaene, 1999; Lee, 2000). The dissociation between subtraction and multiplication in SD is therefore consistent with the notion of a specific impairment of the verbal number code in this condition. We examined whether this pattern was maintained for MK at a late-stage of the disorder.

\subsection{Method}

At 4 years post onset, MK was asked to solve 18 singledigit calculations, presented in Arabic numerals. The calculations were divided equally between the operations of addition, subtraction and multiplication, presented in a mixed fashion (division was not tested due to MK's poor performance for this operation at an earlier stage of the disease). The particular operation required on each trial was indicated by the standard symbols, ' + ', ' - ' and ' $x$ '. The meaning of these symbols was explained to MK prior to testing and it proved necessary to provide repeated reminders throughout the test. MK had previously attempted 14 of these calculations at 2 years post onset, as part of a larger set of problems that included multi-digit calculations and division. These data have been published elsewhere (Jefferies et al., 2004b) and will not be considered in detail here; in brief, MK showed the expected pattern of good addition and subtraction, poorer performance on multiplication and division questions and a strong effect of operand size.

\subsection{Results}

MK showed a considerable decline in calculation performance. Her accuracy fell from $86 \%$ trials correct at 2 years post onset to $36 \%$ correct at 4 years post onset (including only those trials that she was tested on twice; McNemar Test: $p<0.05)$. At a late-stage of the condition, she was still able to perform simple addition (6/6 trials correct), but her subtraction and multiplication performance was poor $(2 / 6$ and $0 / 6$ trials, respectively). She appeared to have difficulty understanding the mathematical symbols that specified the required operation: indeed, 9/10 of her errors arose because she performed addition rather than subtraction or multiplication with the operands (e.g., $2 \times 4=6$ ). In an attempt to facilitate MK's comprehension of the task, the subtraction and multiplication problems were re-presented in a blocked fashion. The required operation was demonstrated for some practice sums. With this support, MK showed better performance for subtraction ( $5 / 6$ trials correct) but she remained unable to perform multiplication (0/6). On some addition and subtraction trials, MK produced a correct spoken response but showed difficulties in writing down her answers as Arabic numerals (these are counted as correct above). This is further evidence that MK's production of Arabic numbers was impaired in an entirely non-verbal task.

\subsection{Discussion}

The pattern of poor multiplication and more intact addition/subtraction persisted at follow up, consistent with the notion of a verbal arithmetic fact deficit in SD. There are several other possible explanations of this finding, however. First, the multiplication questions involved numerically larger responses than the subtractions (although not the additions): this is critical because MK was poor at producing multidigit numbers. In addition, MK's multiplication deficit might have resulted from an impaired understanding of arithmetic signs (as reported by Ferro \& Botelho, 1980). Indeed, her tendency to incorrectly add the operands for multiplication is perhaps suggestive of such a deficit. It is also possible that MK's multiplication difficulties were compounded by procedural difficulties (which have been observed previously in SD; Cappelletti et al., 2001) or poor conceptual knowledge of multiplication (see Delazer \& Benke, 1997; HittmairDelazer, Semenza, \& Denes, 1994). If addition is acquired earlier and performed more frequently than multiplication, it may be more resistant to impairments of this nature. 


\section{Clocks and money}

As noted in the Introduction, anecdotal evidence suggests that SD patients may be able to tell the time and to use money until a late stage of the condition. We devised some explicit tests of MK's abilities in these domains at three to four years post onset. We examined both MK's core understanding of money/time (by asking, for example, which of two coins is worth more) and her ability to generate and comprehend the names of coins/notes and times; these latter tasks might be expected to decline in SD in line with the impairment of the verbal number code documented above.

\subsection{Money}

MK was shown all of the coins and notes up to a value of $£ 20$. There were eight coins $(1 \mathrm{p}, 2 \mathrm{p}, 5 \mathrm{p}, 10 \mathrm{p}, 20 \mathrm{p}, 50 \mathrm{p}$, $£ 1, £ 2)$ and three notes $(£ 5, £ 10, £ 20)$. Their values in Arabic numerals and English were covered over, and the words "pounds" and "pence" were also concealed. MK was shown the items one at a time and asked to name them. She provided the correct number for the coins and notes in 6/11 trials but she almost never included the word "pounds" or "pence" (i.e., $10 \mathrm{p}$ and $£ 10$ were both called "ten"). Her errors suggested some confusion of the words $1 / 10,2 / 20$ and 5/15/50 (e.g., $1 \mathrm{p} \rightarrow$ "a little ten"; $£ 2 \rightarrow$ "twenty").

MK performed poorly in a word-picture matching task using the same items $(2 / 11)$. All the coins and notes were set out in front of her and she was asked to point to each one in turn. Many of her errors suggested a failure to comprehend the words "pounds" and "pence" and an inability to distinguish between words like two/twenty (for example, she selected the $£ 2$ coin instead of the $£ 20$ note). Nevertheless, she performed better than chance when the items were presented in pairs and she was asked which one was worth more (14/20; exact probability on binomial test, $p<0.05$ ). Her ability to determine the monetary value of notes and coins may have relied on the weight/size of the items, however.

\subsection{Clocks}

MK was shown 12 clock pictures and was asked to tell the time. The pictures depicted the minute/hour hands as long/short arrows and the clock face as a ring of Arabic numbers within a circle. MK produced the correct number on $4 / 4$ "o'clock" trials which depicted an exact hour (e.g., she said "three" for 3 o'clock). Her responses to other times, including quarter past the hour (two trials), half past the hour (two trials) and quarter to the hour (two trials), typically consisted of reading the numbers that the arrows pointed to: for example, half past one $\rightarrow$ "one, six" and quarter past four $\rightarrow$ "four, three". On some trials she correctly produced the word "quarter" (e.g., quarter to three $\rightarrow$ "quarter three on the nine"). She showed a strong tendency to produce the number corresponding to the hour before that of the minute hand, suggesting that although her responses did not embody conventional syntax, they may have followed a meaningful pattern. Her number production in this task never went beyond the Arabic numerals present on the clock face; for example, twenty to one $\rightarrow$ "one up to eight" (the word twenty was not included).

MK was shown all 12 clock pictures together and was asked to point to the one that represented a specific time. She only scored 5/12 in this word-picture matching task, although this level of performance was significantly better than chance (binomial $p<0.01$, assuming that $P($ correct $)=0.083$ throughout the test). MK also showed some ability to draw the time to dictation but only for 'o'clock' times (5/6 trials correct). In this task, she was given a clock template and was asked to draw the hands. She did not respond correctly for other times ( $0 / 3$ trials). We attempted to explore MK's core understanding of clocks/time in a task equivalent to magnitude comparison (she was shown two clocks and was asked which is earlier in the day), but she was unable to comprehend this task.

\subsection{Discussion}

MK was able to produce some simple number names for money/clocks but her responses did not embody conventional syntax and her comprehension of these names was impaired in a word-picture matching task. Her understanding of monetary value was also impaired, although not at chance.

\section{Opposites}

We examined MK's comprehension of non-number words that had a dimensional meaning-for example, up/down, big/small. These 'opposites' words may, like number words, derive some protection from the unimpaired parietal magnitude system: insight into their dimensional nature may be retained even if their precise meaning is degraded. This suggestion is consistent with the view that space and number processing draws on the same underlying magnitude code (Dehaene et al., 1998; Walsh, 2003).

\subsection{Method}

MK was asked to pick the opposite of words like 'young', 'fast', and 'short' from a choice of three. In one version of the test, the distracters were all opposites words: e.g., "what is the opposite of 'fat' - high, young or thin?" This test should be impaired if the meanings of different dimensional words are confused (e.g., if "fat" is confused with words such as "tall"). In a second version of the test, the distracter words were replaced by non-opposites items: e.g., "what is the opposite of 'fat' - course, group or thin?" Success at this test requires an understanding of the dimensionality of opposites but not their specific meanings. A third version of the test probed MK's understanding of non-opposites words. She was asked to pick the word related in meaning to the target (e.g., "which word is related in meaning to 'uncle'-school, room or cousin?"). 
The non-opposites words in the second and third versions of the test were matched to the opposites they replaced on an item-by-item basis for word frequency, imageability and syllable length, using data from the MRC psycholinguistic database (Coltheart, 1981). There were 20 trials in each test. The items were presented as both spoken and written words. The correct choice was positioned as the first, second and third option equally often.

\subsection{Results}

MK did poorly in the first version of the test, when all the words were opposites $(6 / 20$ trials correct: no better than chance on binomial test). This test had previously been administered to another late-stage SD patient, FM, who also performed poorly, scoring 12/20 when first tested and only $8 / 20$ a year later (Karalyn Patterson, personal communication). MK did rather better in the second version of the test when the distracters were non-opposites words (12/20: better than chance on binomial test, $p<0.001$ ), suggesting that while the exact meanings of opposites words had become degraded, MK may have retained some understanding of them. MK performed poorly on the task involving non-opposites words ( $8 / 20$ trials correct: no better than chance on binomial test). The accuracy difference between the first and second versions of the test approached significance (Fischer's exact test, $p=0.06$ ).

\subsection{Discussion}

These data suggest that while MK's understanding of opposites words was impaired (i.e., she showed a specific confusion of words like "fat" and "tall"), she may have retained an understanding of their dimensional nature. We acknowledge, however, that an alternative possibility exists: namely that a sensitivity to part of speech underpinned MK's better performance on items such as "what is the opposite of 'fat'-course, group or thin?"

\section{General discussion}

This late-stage SD patient MK showed marked difficulties in tasks tapping the verbal number code but demonstrated relatively intact understanding of numerical magnitude when tested with non-symbolic or Arabic number formats. Previous studies have demonstrated that many aspects of number knowledge remain unimpaired in SD (Cappelletti et al., 2001; Crutch \& Warrington, 2002; Diesfeldt, 1993; Jefferies et al., 2004b). This category advantage is thought to arise because the disease spares the parietal lobe magnitude system critical for number processing. Indeed, patient MK's continued understanding of numerical magnitude in late-stage SD provides further evidence for the separation of at least some aspects of number semantics from the temporal lobe semantic system. Models of numerical cognition that favour a separation between verbal and magnitude representations of number (Dehaene \& Cohen, 1995) might, however, predict a restricted impairment of the verbal number code in SD (e.g., Lemer et al., 2003). Our results provide explicit support for this hypothesis. Although number words were largely unimpaired at an early stage of the disease in line with previous reports (presumably because of their association with an intact representation of numerical magnitude), at a later stage, domain-general semantic decline produced a substantial impairment of the verbal number code, despite MK's good comprehension of magnitude. This finding suggests that the temporal lobe semantic system may contribute to the meanings of number words by representing associations between verbal numbers and Arabic numerals/magnitudes that pertain to the same quantity. The potential role of this system has been largely ignored in anatomical and functional models of numerical cognition (see Dehaene \& Cohen, 1995).

MK had difficulty on a variety of tasks that required the production or comprehension of number words and showed substantial decline in these abilities over the course of the study:

(1) She was poor at transcoding between English number words and Arabic numbers.

(2) She was impaired at naming dot arrays and was poor at word-picture matching with these stimuli. She used a counting strategy to name multi-digit number words although her performance was characterised by miscounts.

(3) She showed a decline in reading aloud irregularly spelled number words. Patients with mild to moderate SD, who retain a good understanding of number words, typically show better reading of number than non-number words. It is argued that this finding reflects the role of the semantic system in reading aloud (Butterworth et al., 2001). The lessening of this effect for MK is consistent with the notion that her lexical-semantic representations of numbers became degraded.

(4) She began to make frequent phonological errors in digit span as her comprehension and production of single-digit number words declined. This finding is consistent with the theory that lexical-semantic representations make an important contribution to the coherence of phonological representations in verbal STM tasks (Patterson et al., 1994).

(5) MK showed excellent performance on a magnitude comparison task involving Arabic numbers. In contrast, she was poor at this task when it was presented using English number words. She showed particularly poor performance for pairs such as "seventeen" and "seventy". These number words should be easily distinguishable in an analogue magnitude code but are derived from a single lexical item, and might become confused in the context of domain-general lexical-semantic decline.

(6) She was able to arrange numbers in order when they were presented as dot pictures or Arabic numerals, but not when they were presented as number words. 
(7) She could still perform simple addition and subtraction. In contrast, she was unable to solve multiplication problems. Patients with less severe SD also show a similar dissociation between intact addition/subtraction and impaired multiplication (Cappelletti et al., 2001; Jefferies et al., 2004b; Lemer et al., 2003), consistent with the notion that multiplication problems are typically solved by accessing a table of memorised facts stored in a verbal code. Poor comprehension of the mathematical symbols may also have contributed to MK's multiplication impairment.

MK showed a clear dissociation between her knowledge of numerical magnitude, which was preserved even at the end of the study, and the verbal number code, which became impaired, consistent with the distinction made by Dehaene and Cohen (1995) in their 'triple code' model. Although one previous study was suggestive of a mild specific impairment to the verbal number code in SD (Lemer et al., 2003), our investigation demonstrates that a wide range of verbal number tasks can become impaired at a late-stage of this condition. It is important to note that non-numerical task demands were not perfectly equated between the verbal and non-verbal number tests. MK performed well in a two-alternative forced choice magnitude comparison task, but rather less well in several tasks that involved the spontaneous production or unguided comprehension of English number words (e.g., transcoding with Arabic numbers and dot arrays, number word reading). She also showed poor performance in wordpicture matching between spoken number words and dot arrays: although this task required a choice among alternatives, the number of possible responses was substantially larger. Nevertheless, these differing task demands cannot account for the complete pattern of performance observed here. In particular, MK was more accurate at magnitude comparison and number sequencing tests when the stimuli were presented as Arabic numerals and dot arrays, compared with number words.

The temporal lobe semantic system, which degrades in $\mathrm{SD}$, is thought to acquire multi-modal mappings between different sensory inputs: e.g., visual and verbal (Rogers et al., 2004a). By extension, this system might be expected to play a role in binding together different number formats that pertain to a single magnitude, by forming mappings between the magnitude, verbal and visual number codes. MK was poor at producing number words/Arabic numerals in response to non-symbolic representations of magnitude, and at transcoding between English and Arabic number formats, in line with this hypothesis. This purported temporal lobe "semantic" number representation is distinct from the "semantic" representation of number quantity that is incorporated in various models of number processing (e.g., McCloskey et al., 1985).

This suggested role for the temporal lobe semantic system in number processing anticipates that late-stage SD patients should have increasing difficulty activating appropriate mag- nitude representations for both verbal and Arabic numbers (in contrast, processing of non-symbolic numbers should remain intact). Although MK showed clear deficits in comprehending verbal numbers, evidence for an impairment of Arabic numbers was equivocal. MK became impaired at producing Arabic numerals in response to both spoken number words and dot pictures; however, she remained able to match these symbols to dot arrays, place them in the correct sequence and judge their relative magnitude. Tasks requiring production are likely to be particularly sensitive to impairment; nevertheless, across a range of tasks (transcoding, sequencing and magnitude comparison), MK performed more poorly for verbal numbers than Arabic numbers. This discrepancy between number formats may have been a consequence of the lateralisation of MK's atrophy, which was more marked in the left hemisphere. According to some viewpoints, both verbal and non-verbal knowledge is distributed across a unitary bilateral semantic system but the left hemisphere has stronger connections to phonology (Lambon Ralph et al., 2001) - consequently, left hemisphere lesions might be expected to produce greater deficits for number words than Arabic numerals. In the number domain, research suggests that although both hemispheres can access the magnitude of Arabic numbers, the left hemisphere plays a unique role in reading aloud numbers (Cohen \& Dehaene, 1995a, 1996). In addition, there are connections between Arabic numerals and their associated magnitudes which are not verbally mediated (e.g., Cipolotti \& Butterworth, 1995; Cohen \& Dehaene, 1995b; Dehaene et al., 1993; Delazer \& Bartha, 2001; Fischer et al., 2003; Whalen et al., 2002). A specific impairment of the verbal number code would therefore allow to access to numerical magnitude from Arabic numbers.

Although the temporal lobe semantic system is thought to be crucial for linking information together across different representational formats (e.g., visual and verbal; Rogers et al., 2004a), semantic degradation might also impact on lexical/visual object representations per se because of interactivity between connected systems. For example, according to one view of the relationship between semantics and phonology in which there are distinct semantic and phonological representations but no separable lexical representations (Patterson et al., 1994; Plaut \& Kello, 1999), lexical knowledge occurs both within the phonological system and in the connections between semantics and phonology, which place additional constraints on phonological processing. Semantic degradation therefore impairs lexical knowledge: consequently, the lexical representations of numerals, as well as the connections between the verbal number code and other formats, may degrade in late-stage SD. Our findings for MK are consistent with this view. A similar argument can be made for visual stimuli (and potentially extended to Arabic numbers): semantic damage not only impairs the ability to name visual stimuli but also disrupts their recognition as visual objects (see Rogers, Lambon Ralph, Hodges, \& Patterson, 2004b). The finding that visual as well as verbal processing is impaired in SD suggests that a central semantic impair- 
ment, rather than two independent deficits of phonological and visual forms, underlies both of these difficulties. In addition to MK's problems with verbal material, she showed the expected pattern of poor object recognition at 3 years post onset (Rogers et al., 2004b). The impact of semantic degradation on verbal/visual processing might have contributed to MK's difficulties in comprehending/producing verbal numbers and her clear deficits in writing Arabic numbers. To summarise, we suggest that MK's severe impairment of temporal lobe semantics was multimodal in that it affected both verbal and visual tasks-in line with this, her processing of both verbal and Arabic numbers was impaired, although her difficulties were more marked for verbal numbers, reflecting her left-lateralised atrophy. Her semantic impairment affected her ability to map between the different number formats and may have also impinged on her lexical knowledge of number and other words.

Several other patients have presented with a pattern of deficits similar to that described here (i.e., intact understanding of numerosity in tasks such as magnitude comparison, poor transcoding between Arabic numerals and English number words and better addition/subtraction than multiplication: Blanken, Dorn, \& Sinn, 1997; Cipolotti \& Butterworth, 1995; Rossor et al., 1995; Verstichel \& Masson, 2003), supporting the view that the magnitude and verbal number codes are dissociable. These patients are typically also aphasic for other classes of words. It is interesting to note that damage to the perisylvian language areas can produce impairments of number processing that are similar to those observed in late-stage SD patients. Cohen et al. (2000), for example, described a patient (ATH) with perisylvian damage who, like MK, was impaired at all tasks involving verbal numbers (e.g., reading aloud, writing to dictation), but had relatively preserved understanding of magnitude, revealed by good performance on number comparison and number bisection tasks. However, ATH could perform magnitude comparison with number words as long as word order/syntax was not critical, whereas MK's performance was poor even for single digit items.

Despite these broad commonalities between patients, our results are unique in showing that degradation of the temporal lobe semantic system can produce a clear dissociation between the magnitude and verbal number codes. Previous reports of number processing in SD have only revealed subtle deficits. Although patients with SD have been shown to be poorer at multiplication than subtraction (Cappelletti et al., 2001; Jefferies et al., 2004b; Lemer et al., 2003), some of their difficulties could have resulted from a loss of procedural knowledge pertaining to multiplication or poor comprehension of arithmetic signs (Cappelletti et al., 2001; Jefferies et al., 2004b), and are not necessarily indicative of an impairment of the verbal number code. One SD patient also showed slower counting of large numbers (Lemer et al., 2003), although she did not show inaccurate counting. By examining a late-stage patient in detail, we have provided more decisive evidence that number words but not their corresponding mag- nitudes degrade in SD. It will clearly be important in future work to establish whether the profile described here is typical of late-stage SD.

\section{Acknowledgements}

We would like to thank Karalyn Patterson for allowing us to use the opposites test and for making some previous data on this test available to us. Thanks are also due to Manabu Ikeda for providing us with MK's initial calculation data. The first author was supported in part by an ESRC studentship. The work was also supported by a grant from the NIMH (MH64445).

\section{References}

Alameda, J. R., Cuetos, F., \& Brysbaert, M. (2003). The number 747 is named faster after seeing Boeing than after seeing Levi's: Associative priming in the processing of multidigit Arabic numerals. Quarterly Journal of Experimental Psychology, 56A, 1009-1019.

Anderson, S. W., Damasio, A. R., \& Damasio, H. (1990). Troubled letters but not numbers: Domain specific cognitive impairments following focal damage in frontal cortex. Brain, 113, 749-766.

Ashcraft, M. H. (1992). Cognitive arithmetic: A review of data and theory. Cognition, 44, 75-106.

Blanken, G., Dorn, M., \& Sinn, H. (1997). Inversion errors in Arabic number reading: Is there a non-semantic route. Brain and Cognition, 4, 404-423.

Bozeat, S., Lambon Ralph, M. A., Patterson, K., Garrard, P., \& Hodges, J. R. (2000). Non-verbal semantic impairment in semantic dementia. Neuropsychologia, 38, 1207-1215.

Bozeat, S., Lambon Ralph, M. A., Patterson, K., \& Hodges, J. R. (2002). The influence of personal familiarity and context on object use in semantic dementia. Neurocase, 8, 127-134.

Butterworth, B., Cappelletti, M., \& Kopelman, M. (2001). Category specificity in reading and writing: The case of number words. Nature Neuroscience, 4, 784-786.

Cappelletti, M., Butterworth, B., \& Kopelman, M. (2001). Spared numerical abilities in a case of semantic dementia. Neuropsychologia, 39, 1224-1239.

Cappelletti, M., Kopelman, M., \& Butterworth, B. (2002). Why semantic dementia drives you to the dogs (but not to the horses): A theoretical account. Cognitive Neuropsychology, 19, 483-503.

Chochon, F., Cohen, L., Van de Moortele, P. F., \& Dehaene, S. (1999). Differential contributions of the left and right inferior parietal lobules to number processing. Journal of Cognitive Neuroscience, 11, $617-630$.

Cipolotti, L., \& Butterworth, B. (1995). Towards a multiroute model of number processing: Impaired transcoding with impaired calculation skills. Journal of Experimental Psychology: General, 124, 375-390.

Cipolotti, L., Butterworth, B., \& Denes, G. (1991). A specific deficit for numbers in a case of dense acalculia. Brain, 114, 2619-2637.

Cohen, L., \& Dehaene, S. (1995a). Number processing in pure alexia: The effect of hemispheric asymmetries and task demands. Neurocase, 1, 121-137.

Cohen, L., \& Dehaene, S. (1995b). Reading numbers in pure alexia: Effects of the task and hemispheric specialisation. Revue Neurologique, 151, 480-485.

Cohen, L., \& Dehaene, S. (1996). Cerebral networks for number processing: Evidence from a case of posterior callosal lesion. Neurocase, 2, $155-174$. 
Cohen, L., Dehaene, S., \& Verstichel, P. (1994). Number words and number non-words: A case of deep dyslexia extending to Arabic numerals. Brain, 117, 267-279.

Cohen, L., Dehaene, S., Chochon, F., Lehericy, S., \& Naccache, L. (2000). Language and calculation within the parietal lobe: A combined cognitive, anatomical and fMRI study. Neuropsychologia, 38, 1426-1440.

Coltheart, M. (1981). The MRC psycholinguistic database. Quarterly Journal of Experimental Psychology, 33A, 497-505.

Crutch, S. J., \& Warrington, E. K. (2002). Preserved calculation skills in a case of semantic dementia. Cortex, 38, 389-399.

Dehaene, S. (1989). The psychophysics of numerical comparison: A reexamination of apparently incompatible data. Perception and Psychophysics, 45, 557-566.

Dehaene, S. (1992). Varieties of numerical abilities. Cognition, 44, 1-42.

Dehaene, S., \& Cohen, L. (1995). Towards an anatomical and functional model of numerical processing. Mathematical Cognition, 1, 83-120.

Dehaene, S., \& Cohen, L. (1997). Cerebral pathways for calculation: Double dissociation between rote verbal and quantitative knowledge of arithmetic. Cortex, 33, 219-250.

Dehaene, S., Dupoux, E., \& Mehler, J. (1990). Is numerical comparison digital? Analogical and symbolic effects in two-digit number comparison. Journal of Experimental Psychology: Human Perception and Performance, 16, 626-641.

Dehaene, S., Bossini, S., \& Giraux, P. (1993). The mental representation of parity and number magnitude. Journal of Experimental Psychology: General, 122, 371-396.

Dehaene, S., Tzourio, N., Frak, V., Raynaud, L., Cohen, L., \& Mehler, J. (1996). Cerebral activations during number multiplication and comparison: A PET study. Neuropsychologia, 34, 1097-1106.

Dehaene, S., Dehaene-Lambertz, G., \& Cohen, L. (1998). Abstract representations of numbers in the animal and human brain. Trends in Neuroscience, 21, 355-361.

Dehaene, S., Spelke, E., Pinel, P., Stanescu, R., \& Tsivkin, S. (1999). Sources of mathematical thinking: Behavioral and brain-imaging evidence. Science, 284, 970-974.

Dehaene, S., Piazza, M., Pinel, P., \& Cohen, L. (2003). Three parietal ciruits for number processing. Cognitive Neuropsychology, 20, 487-506.

Delazer, M., \& Bartha, L. (2001). Transcoding and calculation in aphasia. Aphasiology, 15, 649-679.

Delazer, M., \& Benke, T. (1997). Arithmetic facts without meaning. Cortex, 33, 697-710.

Delazer, M., \& Butterworth, B. (1997). A dissociation of number meanings. Cognitive Neuropsychology, 14, 613-636.

Delazer, M., \& Girelli, L. (1997). When 'Alfa Romeo' facilitates 164: Semantic effects in verbal number production. Neurocase, 3, 461-475.

Deloche, G., \& Seron, X. (1982a). From one to 1: An analysis of a transcoding process by means of neuropsychological data. Cognition, 12, 119-149.

Deloche, G., \& Seron, X. (1982b). From three to 3: A differential analysis of skills in transcoding quantities between patients with Broca's and Wernicke's aphasia. Brain, 105, 719-733.

Diesfeldt, H. F. A. (1993). Progressive decline of semantic memory with preservation of number processing and calculation. Behavioural Neurology, 6, 239-242.

Ferro, J. M., \& Botelho, S. (1980). Alexia for arithmetical signs: A cause of disturbed calculation. Cortex, 16, 175-180.

Fischer, M. H., Castel, A. D., Dodd, M. D., \& Pratt, J. (2003). Perceiving numbers causes spatial shifts of attention. Nature Neuroscience, 6 , 555-556.

Folstein, M. F., Folstein, S. E., \& McHugh, P. R. (1975). Mini-mental state: A practical method for grading the cognitive state of patients for the clinician. Journal of Psychiatric Research, 12, 189-198.

Funnell, E. (1995). Objects and properties: A study of the breakdown of semantic memory. Memory, 3, 497-518.

Galton, C. J., Patterson, K., Graham, K., Lambon Ralph, M. A., Williams, G., Antoun, N., et al. (2001). Differing patterns of temporal atrophy in Alzheimer's disease and semantic dementia. Neurology, 57, 216225.

Graham, N. L., Patterson, K., \& Hodges, J. R. (2001). The emergence of jargon in progressive fluent dysgraphia: The widening gap between target and response. Cognitive Neuropsychology, 18, 343-361.

Hittmair-Delazer, M., Semenza, C., \& Denes, G. (1994). Concepts and facts in calculation. Brain, 117, 715-728.

Hodges, J. R., \& Patterson, K. (1995). Is semantic memory consistently impaired early in the course of Alzheimer's disease? Neuroanatomical and diagnostic implications. Neuropsychologia, 33, 441-459.

Hodges, J. R., Patterson, K., Oxbury, S., \& Funnell, E. (1992). Semantic dementia: Progressive fluent aphasia with temporal lobe atrophy. Brain, 115, 1783-1806.

Howard, D., \& Patterson, K. (1992). Pyramids and palm trees: A test of semantic access from pictures and words. Bury St. Edmunds, Suffolk: Thames Valley Test Company.

Jefferies, E., Jones, R., Bateman, D., \& Lambon Ralph, M. A. (2004). When does word meaning affect immediate serial recall in semantic dementia? Cognitive, Affective and Behavioral Neuroscience, 20, $20-42$.

Jefferies, E., Patterson, K., Jones, R. W., Bateman, D., \& Lambon Ralph, M. A. (2004). A category-specific advantage for numbers in verbal short-term memory: Evidence from semantic dementia. Neuropsychologia, 42, 639-660.

Jefferies, E., Jones, R., Bateman, D., \& Lambon Ralph, M. A. (in press). A semantic contribution to nonword recall? Evidence for intact phonological processes in semantic dementia. Cognitive Neuropsychology.

Jefferies, E., Frankish, C. R., \& Lambon Ralph, M. A. (submitted-a). Lexical and semantic binding in short-term memory: Evidence from normal recall and semantic dementia.

Jefferies, E., Patterson, K., Bateman, D., Jones, R., \& Lambon Ralph, M. A. (submitted-b). The natural history of late-stage "pure" semantic dementia.

Kessler, J., \& Kalbe, E. (1996). Written numeral transcoding in patients with Alzheimer's disease. Cortex, 32, 755-761.

Knott, R. (1998). The impact of lexical and semantic impairment on short-term verbal recall. Unpublished thesis, University of Cambridge, Cambridge.

Knott, R., Patterson, K., \& Hodges, J. R. (1997). Lexical and semantic binding effects in short-term memory: Evidence from semantic dementia. Cognitive Neuropsychology, 14, 1165-1216.

Knott, R., Patterson, K., \& Hodges, J. R. (2000). The role of speech production in auditory-verbal short-term memory: Evidence from progressive fluent aphasia. Neuropsychologia, 38, 125-142.

Lambon Ralph, M. A., McClelland, J. L., Patterson, K., Galton, C. J., \& Hodges, J. R. (2001). No right to speak? The relationship between object naming and semantic impairment: Neuropsychological evidence and a computational model. Journal of Cognitive Neuroscience, 13, 341-356.

Lambon Ralph, M. A., Patterson, K., Garrard, P., \& Hodges, J. R. (2003). Semantic dementia with category specificity: A comparative caseseries study. Cognitive Neuropsychology, 20, 307-326.

Lee, K. M. (2000). Cortical areas differentially involved in multiplication and subtraction: A functional magnetic resonance imaging study and correlation with a case of selective acalculia. Annals of Neurology, 48, 657-661.

Lemer, C., Dehaene, S., Spelke, E., \& Cohen, L. (2003). Approximate quantities and exact number words: Dissociable systems. Neuropsychologia, 41, 1942-1958.

Lezak, M. (1976). Neuropsychological assessment. New York: Oxford University Press.

Martin, N., \& Saffran, E. M. (1997). Language and auditory-verbal shortterm memory impairments: Evidence for common underlying processes. Cognitive Neuropsychology., 14, 641-682.

McCloskey, M., Caramazza, A., \& Basili, A. (1985). Cognitive mechanisms in number processing and calculation: Evidence from dyscalculia. Brain and Cognition, 4, 171-196. 
McCloskey, M., Sokol, S. M., \& Goodman, R. A. (1986). Cognitive processes in verbal number production: Inferences from the performance of brain-damaged subjects. Journal of Experimental Psychology: General, 115, 307-330.

Moyer, R. S., \& Landauer, T. K. (1967). Time required for judgements of numerical inequality. Nature, 215, 1519-1520.

Mummery, C. J., Patterson, K., Price, C. J., Ashburner, J., Frackowiak, R. S. J., \& Hodges, J. R. (2000). A voxel-based morphometry study of semantic dementia: Relationship between temporal lobe atrophy and semantic memory. Annals of Neurology, 47, 36-45.

Noel, M. P., \& Seron, X. (1997). On the existence of intermediate representations. Journal of Experimental Psychology: Learning, Memory and Cognition, 23, 697-720.

Patterson, K., \& Hodges, J. R. (1992). Deterioration of word meaning: Implications for reading. Neuropsychologia, 30, 1025-1040.

Patterson, K., Graham, N., \& Hodges, J. R. (1994). The impact of semantic memory loss on phonological representations. Journal of Cognitive Neuroscience, 6, 57-69.

Patterson, K., Suzuki, T., Wydell, T., \& Sasanuma, S. (1995). Progressive aphasia and surface alexia in Japanese. Neurocase, 1, 115-165.

Pinel, P., Dehaene, S., Riviere, D., \& LeBihan, D. (2001). Modulation of parietal activation by semantic distance in a number comparison task. Neuroimage, 14, 1013-1026.

Plaut, D. C., \& Kello, C. T. (1999). The emergence of phonology from the interplay of speech comprehension and production: A distributed connectionist approach. In B. MacWhinney (Ed.), The emergence of language. Mahweh, NJ: Erlbaum.

Plaut, D., McClelland, J. L., Seidenberg, M., \& Patterson, K. (1996). Understanding normal and impaired word reading: Computational principles in quasi-regular domains. Psychological Review, 103, 56-115.

Raven, J. C. (1962). Coloured progressive matrices sets $A, A B$, B. London: H.K. Lewis.

Rogers, T. T., Lambon Ralph, M. A., Hodges, J. R., \& Patterson, K. (2003). Object recognition under semantic impairment: The effects of conceptual regularities on perceptual decisions. Language and Cognitive Processes, 18, 625-662.

Rogers, T. T., Lambon Ralph, M. A., Garrard, P., Bozeat, S., McClelland, J. L., Hodges, J. R., et al. (2004). The structure and deterioration of semantic memory: A neuropsychological and computational investigation. Psychological Review, 111, 205-235.

Rogers, T. T., Lambon Ralph, M. A., Hodges, J. R., \& Patterson, K. (2004). Natural selection: The impact of semantic impairment on lexical and object decision. Cognitive Neuropsychology, 21, 331352.

Rossor, N. M., Warrington, E. K., \& Cipolotti, L. (1995). The isolation of calculation skills. Journal of Neurology, 242, 78-81.
Seron, X., \& Deloche, G. (1983). From 4 to four: A supplement from three to 3. Brain, 106, 735-744.

Seron, X., \& Deloche, G. (1984). From 2 to two: An analysis of a transcoding process by means of neuropsychological evidence. Journal of Psycholinguistic Research, 13, 215-235.

Snowden, J. S., Griffiths, H., \& Neary, D. (1994). Semantic dementia: Autobiographical contribution to preservation of meaning. Cognitive Neuropsychology, 11, 265-288.

Snowden, J. S., Griffiths, H. L., \& Neary, D. (1996). Semantic-episodic memory interactions in semantic dementia: Implications for retrograde memory function. Cognitive Neuropsychology, 13, 1101-1137.

Snowden, J. S., Neary, D., \& Mann, D. M. A. (Eds.). (1996). Frontotemporal lobar degeneration: Frontotemporal dementia, progressive aphasia, semantic dementia. London: Churchill Livingstone.

Stanescu-Cosson, R., Pinel, P., van de Moortele, P., Le Bihan, D., Cohen, L., \& Dehaene, S. (2000). Understanding dissociations in dyscalculia: A brain imaging study of the impact of number size on the cerebral networks for exact and approximate calculation. Brain, 123, 2240-2255.

Takayama, Y., Sugishita, M., Akiguchi, I., \& Kimura, J. (1994). Isolated acalculia due to left parietal lesion. Archives of Neurology, 51, 286-291.

Thioux, M., Pillon, A., Samson, D., de Partz, M. P., Noel, M. P., \& Seron, X. (1998). The isolation of numerals at the semantic level. Neurocase, 4, 371-389.

Thioux, M., Ivanoiu, A., Turconi, E., \& Seron, X. (1999). Intrusion of the verbal code during the production of Arbic numerals: A single case study in a patient with probable Alzheimer's disease. Cognitive Neuropsychology, 16, 749-773.

Verstichel, P., \& Masson, C. (2003). Progressive acalculia and impairment in number processing revealing a cerebral degenerative disease. Revue Neurologique, 159, 413-420.

Walsh, V. (2003). A theory of magnitude: Common cortical metrics of time, space and quantity. Trends in Cognitive Sciences, 7, 483-488.

Warrington, E. K. (1975). The selective impairment of semantic memory. The Quarterly Journal of Experimental Psychology, 27, 635-657.

Warrington, E. K., \& James, M. (1991). The visual object and space perception battery. Bury St. Edmunds, Suffolk: Thames Valley Test Company.

Wechsler, D. (1987). Wechsler Memory Scale-Revised (WMS-R). New York: Psychological Corporation.

Wechsler, D. (1997). Wechsler Memory Scale-WMS-III (3rd ed). The Psychological Corporation, London.

Whalen, J., McCloskey, M., Lindemann, M., \& Bouton, G. (2002). Representing arithmetic table facts in memory: Evidence from acquired impairments. Cognitive Neuropsychology, 19, 505-522. 